\title{
NUOVE NOTIZIE SUI RAPPORTI ECONOMICO-POLITICI TRA LA REPUBBLICA DI GENOVA E IL GIUDICATO DI ARBOREA FRA TRE E QUATTROCENTO
} $(1387-1410)$

\author{
LUCIANo GaLlinaRI \\ Istituto sui Rapporti Italo-Iberici \\ (CNR, Cagliari, Italia)
}

\begin{abstract}
Recenti ricerche archivistiche hanno permesso di rinvenire nuovi documenti sulle relazioni politiche ed economiche intercorse tra la Corona d'Aragona, il Comune di Genova e il regno di Arborea fra Tre e Quattrocento, quando la famiglia Doria, una delle più importanti consorterie liguri, ben presente anche in Sardegna, gestl direttamente nella quasi totalita i rapporti politici ed economici tra il Comune e il Giudicato, data l'influenza esercitata a Genova e la sua contemporanea unione con la famiglia regnante di Oristano realizzatasi con il matrimonio tra Eleonora di Arborea e Brancaleone Doria, dal quale nacquero Federico e Mariano Doria-Bas che per alcuni anni incarnarono l'unità dei destini delle due grandi Case ${ }^{1}$.

Soffermarsi sulla politica della famiglia Doria, volta non a costituire un semplice insieme di feudi e possedimenti territoriali ma un vero e proprio stato coloniale frutto di imprese di conquista e di alleanze politiche,
\end{abstract}

\footnotetext{
'Cfr. G. A. ASCHERI, Notizie storiche intomo alla riunione delle famiglie in alberghi in Genova, Genova, 1846; Genealogie medioevali di Sardegna, a cura di L. L. Brook-F. C. Casula-M. M. Costa-A. M. Oliva-R.Pavoni-M. Tangheroni, Sessari, 1984, tav. XXI, lemma 29: G. PETTI BALBI. Strutture familiari nella Liguria medievale, in "Alti del Congresso 'I Luguri dall'Amo all 'Ebro' in ricordo di Nino Lamboglia", III, Bordighera, 1984, p. 79, e J. HI:liks, Genova nel 400, Milano, 1991, pp. 335-341, e P. F. SimbULA, Casteldoria dote matrimoniale di Eleonora d'Arborea, "Medioevo. Saggi e Rassegne", 16 (1991), pp. 117-134.
} 
vivificato da un'economia di mercato, permette di comprendere meglio il suo ruolo di tenace oppositrice alla conquista catalana della Sardegna ${ }^{2}$.

La documentazione esaminata ci porta a ritenere che in questo periodo l'isola costitul un problema che Genova non poté o non volle seguire in modo diretto e che pertanto, considerata la antica e consolidata presenza dei Doria nell'isola, abbia quasi delegato ad essi la cura degli interessi liguri, intervenendo sempre in maniera più o meno indiretta e ufficiosa per non alimentare ulteriori urti politici e militari con la Corona d'Aragona, legittima detentrice dell'isola a seguito dell'investitura pontificia $^{3}$. Cosi facendo, la Repubblica applico nella seconda metà del '300 anche in Sardegna l'abituale politica coloniale volta, soprattutto per motivi economici, a lasciare l'iniziativa nel campo dei possedimenti d'oltremare sempre più nelle mani dei privati.

Dopo una breve parentesi di relativo allontanamento susseguente alla pace del 1378 con l'Aragona e agli impegni bellici della guerra di Chioggia, vi fu una ripresa della partecipazione diretta di Genova negli affari sardi dai primi anni del regno della giudicessa Eleonora, succeduta al fratello Ugone III nel $1383^{4}$.

In questi anni nel rapporto tra il Giudicato e il Comune scorgiamo un duplice binario: da una parte i rapporti politici -gestiti dai Doria sardi e genovesi- finalizzati a far rimettere piede in Sardegna a Genova mediante una rete di ufficiali che avrebbero dovuto gestire castelli e ville strappati agli aragonesi dai sardi, in cambio degli aiuti forniti a questi ultimi; e

${ }^{2} \mathrm{Cfr}$. G. PETn BALBI, Castelsardo e $i$ Doria all'inizio del secolo XIV, in "Archivio Storico Sardo", XXX, 1976, pp. 189-194, J. HEERS, Pisani e genovesi nella Sardegna medievale: vita politica e sociale (X-XV secolo), in Il Medioevo dai Giudicati agli aragonesi, Storia dei Sardi e della Sardegna, II, Milano, 1987, pp. 236-237. 77.

${ }^{3}$ F.C. CASUlA, La Sardegna aragonese. 1 La Corona d'Aragona, Sassari, 1990, pp. 61-

'Archivio di Stato di Genova (d'ora in avanti ASG), Antico Comune, Officium Monete, reg. 856, ce. Sv e 16r-v. Nel primo documento del 15 gennaio 1384 si legge la nomina di Giovanni de Corvo di Monleone a castellano di una non precisala fortezza "ad paites Sardinee"; dal secondo, con la sola indienzjone dell'anno, invece apprendiamo l'invio nell'isola di Battista de Carvari per ricoprire l'incarico di podestà dell'importante villa di Osilo nei pressi di Sassari. 
dall'altra parte i rapporti economici che videro i cereali arborensi divenire un prezioso elemento di collegamento tra i due stati ${ }^{5}$.

Su questa stessa falsariga anche il 1386, che si rivelo un anno di intensi contatti tra Oristano e Genova. Ne siamo informati dalle fonti liguri: il 26 maggio, per ordine del doge Antoniotto Adorno, i massari del Comune dovettero pagare a Domenico de Prementorio 3 lire 15 soldi e 11 denari restanti dalla somma di 26 lire 10 soldi di Genova, spese dal suddetto quando l'anno precedente venne inviato in Arborea alla presenza della giudicessa Eleonora sulla cocca patronizzata da Gaspare Coccalosa ${ }^{6}$.

Dinanzi al rinsaldarsi dei legami politici ed economici tra Giudicato e Repubblica, la Corona d'Aragona tentò di raggiungere un nuovo accordo diplomatico separato con ognuno di loro.

Con Genova le trattative per una nuova composizione erano già iniziate nel 1385 ed erano state affidate al notaio e cancellerie Antonio Credenza e a Luchino Scarampi, mercante astigiano particolarmente vicino alla corte aragonese. Dalle fonti genovesi risulta che nel febbraio 1386 il suddetto notaio si trovava a Barcellona per proseguire nelle consultazioni con la controparte ${ }^{7}$ La complessità della questione allungava i tempi, al punto

\footnotetext{
${ }^{5} \mathrm{Ai}$ primi di febbraio del 1385 il doge Antoniotto Adorno e il Consiglio degli Anziani della Repubblica decisero d'inviare una galera in Sandegna per l'acquisto di grano, ASG, Antico Comune, Officium Monete, reg. 856, c. 28. Il rinnovato interesse di Genova per il grano sardo secondo M. BALARD, Le commerce du blé en Mer Noire (XIIle-XV sizcles), Aspetti della vita economica medievale. "Atti del Convegno di studi nel $\mathbf{X}$ anniversario della morte di Federigo Melis", Firenze, 1985, pp. 73-74, trae origine dalla contemporanea crisi cerealicola in Levante. Per gli anni immediatamente precendenti J. DAY, Prix agricoles en Méditerranée à la fin du XIV e siècle (1382), in "Annales. Economies, Societés, Civilisations", 16, 1961, p. 636, nota che nei decreti del Consiglio degli Anziani del Comune di Genova del 1381 e 1382 non si rinvengono misure eccezionali per il commercio e l'approvvigionamento di grano, il cui prezzo a Genova riflette fedelmente le condizioni del mercato nel bacino occidentale del Mediterranco.

'ASG, Antico Comune, Magistrorum rationalium appodixiae, reg. 99, c. 41r. Il patrono Gaspare Coccalosa compare anche in altri tre documenti dello stesso registro; il primo a $c$. $61 v$, in data 12 luglio 1386, contenente l'ordine dogale per i massari generali del Comune di pagare al detto patrono la somma di 1500 lire per lo spese di un mese della galera ad custodiam utilizzata per condurre in Oristano Domenico de Prementorio; il secondo a c. 94r, in data 25 ottobre 1386, in cui il doge ordinò ai massari del Comune di pagare al ouddetto Coccalosa 332 lire e 8 denari a complemento dello stipendio dovutogli per la galera patronizzata per cinque mesi e sette giomi dal 1 aprile al 7 settembre di quello stesso anno.

${ }^{7}$ ASG, Antico Comune, magistrorum rationalium appodixiae, reg. 99 , cc. $16 \mathrm{r}$ e $36 \mathrm{r}$. Cfr. G. PISTARINO, Luchino Scarampi tra Genova e Barcellona per la pace del 1386, "Medioevo. Saggi e Rassegne", 1, 1975, pp. 44-47. Il 21 maggio i maestri razionali del Comune autorizzavano il pagamento di 26 lire 7 soldi e 6 denari a Antonio Credenza inviato alla presenza di Pietro IV, come saldo del rimborso delle spese sostenute durante il viaggio.
} 
che sei mesi più tardi il 28 agosto, dopo aver fatto rientro a Genova e aver ricevuto una nuova procura per le trattative, il cancelliere era in procinto di ripartire per Barcellona per concludere i colloqui ${ }^{8}$.

Uno degli argomenti più spinosi affrontati dai plenipotenziari dei due Stati fu quello riguardante gli aiuti economici e militari che Genova continuava a fornire agli arborensi impegnati nella guerra contro i catalanoaragonesi?.

Nonostante tutte le difficoltà incontrate per portare a termine le negoziazioni, tra la fine del 1387 e i primi mesi dell'anno successivo i contatti tra Barcellona e Genova si intensificarono. Finora è stato detto che a spingere maggiormente per la soluzione diplomatica fosse stata la Corona, impegnata militarmente ed economicamente nell'impresa di Sardegna. In realtà dalla documentazione analizzata sembra evincersi che Genova fosse animata ad una conferma degli accordi con l'Aragona soprattutto da motivazioni economiche al cui interno la Sardegna occupava un posto ben determinato ${ }^{10}$.

Il 5 gennaio 1388 da Vilafranca de Penedés Giovanni I comunico a Berenguer de Relat, maestro razionale della sua corte, di aver ricevuto l'ambasciatore inviatogli dalla Repubblica, Andreolo Maniscalco accompa-

\footnotetext{
${ }^{8}$ ASG, Antico Comune, magistrorum rationalium appodixiae, n. 99 , c. $70 \mathrm{r}$.

'Sull'accordo di pace raggiunto il 22 novembre 1386 , ma non ratificato perché di lì a poco il re Pietro IV nurì rimandiamo a L. BALIETTO, Alghero nei trattati fra Genova e l'Aragona (1378-1417), in Genova Mediterraneo e Mar nero (secc. XIII-XV), Civico Istituto Colombiano, Studi e Testi-Serie Storica, 1, Genova, 1976, pp. 26-27, EAD., Questioni del Levante nei trattati tra Genova e l'Aragona dal 1378 al 1417 , in "Segundo Congreso Internacional de Estudios sobre las Culturas del Mediterraneo Occidental", Barcelona, 1978, pp. 239-240, G. MFIoni, Genova e Aragona all'epoca di Pietro il Cerimonioso, Padova, 1982, III, pp. 183. 185 e S. ORIGONE, Dal trattato fra Genova e Sassari (1294) al trattato fra Bonifacio ed Alghero (1386), in "Alti del I Convegno internazionale di studi geografico-storici". La Sardegna nel mondo mediterraneo. 2 Gli aspetti storici. Sassari, 1981, pp. 272-274.

${ }^{10}$ I rapporti commerciali tra Genova o Aragona ebbero uno dei periodi migliori proprio nel ventennio 1370/1390. A conjermarlo sono le medie di viaggi tra operatori cornmerciali dei due Stati: 19 nel 1386; 28 ncl 1392 e nel '93, con una media annuale di 13/14 viaggi, equivalente al doppio (7 viaggi) del biennio 1453..54, chiaro segno della nulevole contrazione dei traffici commerciali tra i due Stati. Cfr. J. HEERS, Il commercio, cit., pp. 187-192 c 207-208. Una situazione di relativa tranquillita con l'Aragona fu ricercata da Genova anche per poter avere maggiore libertà di azione sul mercato francese, parzialmente ripresosi grazie alla lunga tregua con l'Inghilterre all'interno della guerre dei Cent'Anni. Cfr. M. L. CHIAPPA MAURI, Il commercio occidentale di Genova nel XIV secolo, "Nuova Rivista Storica", LVII, 1973, pp. 584591.
} 
gnato da Giuseppe de Grappo, notaio e scriba, i quali partirono da Genova il 22 novembre 1387 e vi fecero ritorno il 15 aprile dell'anno successi$\mathrm{vo}^{11}$.

Dalla lettura di questi documenti emerge una notevole cautela di Giovanni I nell'intavolare trattative diplomatiche con gli ambasciatori della Repubblica ligure, spiegabile considerato l'intensificarsi delle relazioni politiche ed economiche tra Oristano e Genova, in spregio al precedente accordo del 1378 e al trattato di pace con il Giudicato sardo in via di ratifica proprio nei primi giorni del 1388 a Castel di Cagliari ${ }^{12}$.

Ciononostante, le trattative tra la Corte barcellonese e il Comune di Genova proseguirono fino alla ratifica del nuovo trattato di pace nel marzo del $1390^{13}$.

Approfittando del momento di relativa tranquilità nelle relazioni tra la Repubblica e la Corona, i mercanti liguri aumentarono la loro presenza negli scali sardi e in particolare in quello cagliaritano. Come attestano le annotazioni dei registri della serie Aduanas sardas, contenenti le operazioni di acquisto e vendita nelle dogane di Castel di Cagliari, nel 1390 i mercanti liguri importarono ed esportarono diversi prodotti quali vino rosso, formaggi, drappi, cereali (orzo e frumento), coltelli, stoppa, sebo, resina ed altri ancora ${ }^{14}$.

\footnotetext{
"Archivo de la Corona de Aragón (d'ora in poi ACA), Cancilleria, Curiae sigilli secreti, reg. 1952, c. 97v. ASG., Antico Comune, Magistrorum rationalium sententiae; reg. 83, cc. 24v-25r. Altri riferimenti alla missione diplomatica di Andreolo Maniscalco presso la corte aragonese sono contenuti anche in Antico Comune, Communis Janue Massaria, reg. 20, cc. $7 v, 58 v, 208 r$.

${ }^{12}$ Il nuovo accordo venne sottoscritto nella capitale del Regnum Sardiniae da Ximen Peres de Arenòs, governatore generale, e dai plenipotenziari della giudicessa Eleonora, e ratificato dal sovrano 1'8 aprile 1388. Cfr. F. C. CASULA, La storia di Sardegna, Sassari-Pisa, 1992, p. 361.

${ }^{13}$ M. T. FERRER I MALLOL, La pace del 1390 tra la Corona d'Aragona e la Repubblica di Genova, in "Miscellanea di storia ligure in onore di G. Falco", Genova, 1966, p. 162.

${ }^{14} \mathrm{ACA}$, Real Patrimonio, Admanas sardas, reg. $2141 \mathrm{cc} .1 \mathrm{v}, 6 \mathrm{v}, 7 \mathrm{v}, 9 \mathrm{r}-\mathrm{v}, 16 \mathrm{v}, 17 \mathrm{r}, 22 \mathrm{r}$, 38v. Risultano importati dai mercanti della Repubblica nel porto di Castel di Cagliari 171 botti di vino rosso (ca. 77.000 litri), 19 botti e mezza di vino latino ( 8775 litri), oltre a 101 lire di olio, 20 lire di canapa, 22 lire di tele, 9 lire di pelli, 28 lire 15 soldi di panni, 80 lire di cipolle e 80 lire di coltelli. A proposito dell'olio J. DAY, Prix agricoles, cit., p. 644 pone la Sardegna insieme all'Italia del Nord, la Toscana e le citta barbaresche tra le regioni del Mediterraneo occidentale che ne importavano di più, anche se segnala come i clienti preferiti dei mercanti genovesi fossero sempre i paesi levantini. Nel mercato della capitale del Regnum Sardiniae nonostante la precaria stituazione il prezzo dell'olio di oliva si mantenne pressoche costante tra 1369 e 1397, oscillando tra 6 e 7 soldi per quartan (pari 4,1 litri); per cio che riguarda invece il vino, tra 1369 e 1392 il suo prezzo vario tra 2 soldi e denari e 3 soldi e 10
} 
Alcune delle merci esportate risultavano essere d'importanza strategica quali la stoppa e il sebo, fondamentali per l'industria navale, di cui Genova non era particolarmente fornita ${ }^{15}$. Altrettanto interessante si rivela la lieve ripresa delle esportazioni cerealicole nonostante la grave crisi economica che attanagliava il Regnum Sardiniae. I dati offerti dalle fonti doganali cagliaritane permettono di vedere che nel 1390 le esportazioni di orzo ad opera di mercanti genovesi ammontarono a 538 starelli (= 26.470 litri), mentre quelle di frumento furono di soli 32 starelli $(=1.575 \text { litri })^{16}$.

Ben diverse invece le quantità di cereali che gli operatori commerciali liguri poterono acquistare contemporaneamente nel Giudicato d'Arborea. Già Balard aveva calcolato in $\mathbf{3 7 7 2}$ le mine di grano sardo importato a Genova nel 1390, cifra equivalente a quasi 400.000 litri, pari al 5,5\% del totale di cereali importati quell'anno dalla Dominante ${ }^{17}$.

Il fatto che Genova, pur considerando l'elevato costo del prodotto sardo, lo acquistasse conferma la grave crisi di approvvigionamento nelle colonie e nei mercati orientali a partire gia dal 1386 , e ribadisce la necessità per il Comune di avere buone relazioni con la Corona d'Aragona nel bacino occidentale del Mediterraneo ${ }^{18}$.

MancA, Il libro di conti di Miquel Ça-Rovira, Padova, 1969, pp. 113-122.

${ }^{15}$ ACA, Real Patrimonio, Aduanas sardas, reg. 2141 , cc. $42 \mathrm{v}, 44 \mathrm{v}$. In merito al commercio di questi prodotti tra la Repubblica di Genova e la Corona d'Aragona vedasi A. M. ARAGO, Fletes de géneros prohibidos desde el puerto de Barcelona a la Liguria (1358-1409), in "Atti del I congresso storico Liguria-Catalogna", Bordighera, 1974, p. 214.

${ }^{16} \mathrm{ACA}$, Real Patrimonio, Aduanas sardas, reg. 2141 cc. 132r, 172r e 173r. Già M. TANGHeroni, Aspetti del commercio dei cereali nei Paesi della Corona d'Aragona. 1 La Sardegna, Cagliari, 1981, pp. 120-121, evidenziò come nonostante la crisi tra 1389 e 1390 il Regnum giungesse a riesportare cereali, frumento, orzo, sia pure in misura non rilevante, anche se per la seconda metà del secolo appaiono pressoché continue le importazioni cerealicole nell'isola. Dalla lettura dei registri di questa interessante fonte fiscale C. GIORGIONI MERCURIAU, La persistente visalitd del porto di Cagliari nel Trecento: un motivo di riflessione storiografica, in "Atti del secondo convegno intemazionale di studi geografico-storici". $4 \mathrm{La}$ storia del mare e della terra, Sassari, 1984, p. 114-117, ricava che nel corso del XIV secolo la Sardegna commerciava soprattutto con Catalogna, Baleari, Pisa, Genova, Napoli, Gaeta, Tropea, Santonoceto.

"In merito al grano sando M. BALARD, La Romanie genoise (XIle-début du XVe sidcle), "Atti della Società ligure di storia patria", n. s., vol. XVIII (XCII), fasc. II, Genova, 1978, vol. II, pp. $761-762$ e 767 constata che il suo prezzo dopo essere stato al terzo posto durante i mesi estivi di giugno e luglio dopo quelli dei cereali spagnoli e siciliani, ascese decisamente al primo posto nella seconda meta dell'anno.

${ }^{18}$ Le colonie orientali alle prese con pessimi raccolti proibirono l'esportazione dei cereali, come risulta seginalato da diverse lettere di fattori della Compagnia Datini e da un corrispondente dei maestri razionali del Comune. Cfr. M. BALARD, Le commerce, cit., p. 75. 
In questi anni pur con la ripresa delle ostilità sarde contro la Corona, guidate in prima persona da Brancaleone Doria, il mercato cerealicolo arborense fungeva da catalizzatore per il Comune italiano. Nel luglio 1391 risulta acquistata per conto del doge Antoniotto Adorno una partita complessiva di grano sardo ammontante a 1971 mine, pari a circa 208.000 litri da Giovanni dei Signori di Lagneto, esponente di una famiglia dell'albergo Doria in rapporti anche con la dinastia giudicale oristanese, a conferma di come pressoché tutte le relazioni politiche ed economiche tra la Repubblica e il Giudicato passassero attraverso questa consorteria. Sempre nel mese di luglio le fonti liguri segnalano un altro carico di cereali sardi di 1500 mine (158.250 litri), del succitato Lagneto per un totale complessivo di 366.250 litri, che avrebbe dovuto scaricare a Genova o nelle sue Riviere, e di ll avrebbe dovuto far trasportare nella metropoli ${ }^{19}$.

E che il grano acquistato fosse arborense crediamo sia dimostrato almeno da due elementi: innanzitutto da una richiesta del 4 agosto dello stesso anno del governatore generale del Regnum Sardiniae Joan de Montbuy a Brancaleone Doria perché gli concedesse una licenza di esportazione di 700 rasieri di frumento (45.430 litri) da destinare alla spedizione organizzata dal re Giovanni I e dal fratello Martino, duca di Montblanc, per insediare sul trono siciliano Martino il Giovane. In secondo luogo dall'analisi dei registri doganali di Castel di Cagliari che per il 1391 evidenzia come gli operatori commerciali liguri ebbero modo di acquistare nel Regnum Sardiniae solo 432 starelli di orzo, pari a 21.250 litri ${ }^{20}$. Nel frattem-

\footnotetext{
${ }^{19}$ M. BALARD, La Romanir, cit., II, p. 762, stima le 1971 mine citate corrispondenti al 6\% del totale delle importazioni cerealicole della Repubblica. Per il carico supplementare di grano sardo vedasi ASG, Antico Comune, Magistrorum rationalium sententiae, reg. 83, c. 75r. Esso fu portato da Giovanni dei Signori di Lagneto, personaggio che abbiamo incontrato più volte sempre in stretta relazione con il Giudicato d'Arborea e con la famiglia Doria. Diversi anni prima, nel 1382 , aveva operato come testimone al contratto matrimoniale conclu80 tra Bianchina, figlia dell'allora doge di Genova Nicola Guarco, e Federico Doria-Bas figlio di Eleonora e Brancaleone, edito da G. PETTI BALBI, Per la storia dei rapporti tra Genova ed Eleonora d'Arborea, in "Medioevo. Saggi e Rassegne", 9, 1984, p. 41. Sulla famiglia dei Lagneto vedasi R. PAVONI, Signori della Liguria orientale: i Passano e i Lagneto, La Storia dei Genovesi, "Atti del convegno intemazionale di studi sui ceti dirigenti nelle istituzioni di Genova", IX, Genova, 1989, pp. 451484.

${ }^{20}$ Per la richiesta del Montbuy a Brancaleone Doria vedasi ACA, Real Audiencia, Procesos contra los Arborea, IX, cc. $22 \mathrm{r}$-v. Oltre a cio, i mercanti liguri continuarono ad importare a Castel di Cagliari olio, vino rosso, e merci di diverso consumo per il mercato locale come carta, pannilana, tele, stoffe. Sul versante dell'esportazione oltre ai cereali nel 1391 gli operatori commerciali liguri caricarono nelle loro imbarcazioni 256 lire e 10 soldi di formaggi, 7 liro di resina, 12 lire 012 soldi di pelli di daino, 450 litri di vino greco, 9 lire di vino cobertos, came di maialo salata ed altro ancora. ACA, Real Patrimonio, Aduanas Sardas, reg.
} 
po, il 21 settembre 1391 a Giovanni I venne richiesto dal governatore generale di Sardegna Montbuy di scrivere a Genova e Pisa perché smettessero di prestare continui aiuti agli arborensi in spregio ai trattati di pace sottoscritti. Oltre che nei due Comuni italiani, i catalani individuavano le principali basi di rifornimento dei sardi ribelli in Bonifacio e nelle citta provenzali di Nizza e Marsiglia i cui contatti commerciali con il giudicato oristanese erano proseguiti lungo tutto il secolo ${ }^{21}$.

L'aiuto prestato da queste comunità agli arborensi creava grosse difficoltà al sovrano aragonese, il quale oltre a procedere a continue alienazioni di diritti regi e a prestiti dalle diverse comunità della Confederazione, nel Regnum Sardiniae dovette ordinare a tutti i suoi ufficiali di soprassedere e di ritardare la riscossione di tributi, redditi e censi che ogni abitante avrebbe dovuto versare per i successivi 10 anni al baiulo generale ${ }^{22}$.

Ancora più stridente il confronto con il Giudicato sardo che, per quanto provato dai danni e dalle distruzioni della guerra, era ancora in grado di alimentare una discreta corrente esportatrice di cereali verso Genova.

Da diversi conti dell'Officium dei maestri razionali di Genova, in data 31 maggio 1392, veniamo informati dell'armamento di tre galere, una delle quali patronizzata da Pietro Doria, membro di spicco dell'Albergo omonimo, le quali avevano raggiunto Oristano per permettere al comissarius Domenico de Prementorio (o Promontorio) di raggiungere la Corte giudicale"pro factis grani"23.

La missione, svoltasi tra il 21 marzo e il 20 maggio 1392, dovette aver successo: il 9 ottobre il doge Antonio Montaldo stipulo un contratto

2141 , cc. 50r, 96r-v, 98r, 104v, 109v, 139v-140r.

${ }^{21} \mathrm{~F}$. C. CASULA, Carte reali diplomatiche di Giovanni I il cacciatore, re d'Aragona niguardanti l'llalia, Padova, 1977, doc. 28, pp. 56-57. Risulta chiaro che il regno artorense non fosse del tutto isolato sul piano diplomatico come sostenuto a suo tempo da Putzulu, per quanto l'apporto di Provenzali e Pisani non fosse paragonabile a quello offerto da Genova. Cfr. E. PUTZUw, Documenti inediti sul conflitto tra Eleonora d'Arborea e Giovanni I d'Aragona, "Archivio Storico Sardo", XXVII, 1961, p. 96.

${ }^{22}$ ACA, Cancilleria, Curiae sigilli secreti, reg. 1962, cc. 70v-71r, e E. PUTzulu, "Cartulari de Arborea". Raccolta di documenti diplomatici inediti sulle relazioni tra il giudicato d'Arborea e i re d'Aragona (1328-1430), "Archivio Storico Sardo", XXV, fasc. 1-2, 1957, doc. 17, pp. 144-145.

${ }^{23}$ ASG, Antico Comune, Magistrorum rationalium sententiae, reg. 85, cc. 20v-21r. Appena rientrato a Genova il Prementorio presento i conti della sua missione in Sardegna registrati in data del 31 maggio. 
con Antonio Morando, patrono e proprietario con i suoi soci della nave $S$. Maria e S. Giovanni, in base al quale egli si sarebbe dovuto recare a Oristano per caricarvi 6.000 mine di frumento (equivalenti a 633.000 litri), o anche piu se fosse stato possibile, a sua discrezione, da trasportare il più celermente possibile a Genova ${ }^{24}$.

Oltre che dei cereali arborensi, i mercanti liguri non tralasciavano di rifornirsi a Castel di Cagliari di sale, che durante tutta la prima metà del Trecento garantl profitti variabili intorno al 150\%-200\% del capitale investito, e al 700-800 \% per la seconda metà del secolo, allorché aumentarono i rischi per le loro imbarcazioni lungo le coste isolane $e^{25}$.

E' questo un dato di notevole rilevanza per poter meglio comprendere l'atteggiamento tenuto da Genova nel caso sardo. E' certo che la Repubblica non gradi il possesso catalano-aragonese della Sardegna, e che di conseguenza prese fin da subito a fronteggiare la Confederazione iberica aiutando i ribelli sardi. Ma e altrettanto vero che le preoccupazioni maggiori del Comune ligure erano determinate soprattutto dal pericolo che i catalani, una volta stabilmente insediatisi nelle due più grandi isole del Mediterraneo, potessero tagliare a loro piacimento le sue rotte da e per il Levante, dove Genova aveva i suoi interessi vitali, e in cui gli iberici avrebbero potuto intraprendere un ulteriore ondata di stanziamenti.

Dati come quelli poc'anzi citati a proposito del commercio del sale sardo ci rafforzano nella convinzione che se anche Genova non accolse favorevolmente la perdita del proprio status privilegiato negli scali sardi ad opera dei nuovi arrivati catalani, non fu certamente lo spauracchio di un' eventuale estromissione dal mercato isolano a creare ansie e timori alla Superba. Il Comune ligure poté continuare a approvvigionarsi nei porti sardi soprattutto di cereali e sale pur pagando i dazi doganali stabiliti dagli ufficiali regi, che permettevano comunque guadagni niente affatto trascurabili. Un altro elemento va tenuto nella giusta considerazione. Quando i mercanti genovesi in cerca di sale sardo furono presenti nella capitale del Regnum giunsero a movimentare 1 ' $88 \%$ del sale cagliaritano registrato in

\footnotetext{
${ }^{24}$ ASG, Archivio segreto, Diversorum Communis Janue, filzs 1, doc. 72.

${ }^{25} \mathrm{C}$. MANCA, Aspetti dell'espansione economica catalano-aragonese nel Mediterraneo occidentale. Il commercio intemazionale del sale, Biblioteca della rivista "Economia e Storia", 16, Milano, 1965, pp. 209-210.
} 
quegli anni ${ }^{26}$, giungendo a monopolizzare il traffico del sale da esportazione: da 52, 7\% a 71,7\% dei carichi totali nel biennio 1364-1365, alla ripresa delle ostilità tra Mariano IV e l'Aragona; e circa il 66,5\% nel biennio 1391-139227.

Ora, tenendo ben presenti questi dati riteniamo difficile che Genova si sarebbe impegnata in una guerra ad oltranza con la Corona d'Aragona per il possesso della Sardegna. Non per accaparrarsi il restante sale sardo sul quale poteva ricavare gia cosi dei profitti più che ottimi, e nemmeno per i cereali, che la Repubblica poteva pacificamente procurarsi nel Giudicato d'Arborea, i quali invece presero a scarseggiare nel Regnum Sardiniae a partire dalla metà del Trecento tanto da costringere gli stessi catalani a costose operazioni di importazioni di grano, orzo e frumento nell'isola.

Due secoli di storia (XII e XIII) confermano che a Genova non interesso mai un possesso territoriale diretto della Sardegna, lontano dai suoi schemi coloniali, ma solo il controllo del commercio isolano attraverso la sua preponderanza navale ${ }^{23}$. Molto più utile sostenere la ribellione dei giudici oristanesi che impegnava politicamente, militarmente ed economicamente la Confederazione iberica nell'isola distraendola in parte dal piu ricco Levante.

Per quanto importante e strategica nel Mediterraneo occidentale, la Sardegna era solo una piccola tessera dello sconfinato mosaico di Paesi e regioni che costituivano il mercato genovese nel Trecento, estendentesi dall'Oriente all'Atlantico, all'interno del quale i mercanti genovesi avevano rapporti d'affari quotidiani ed intensi con i loro omologhi catalani. Uno scontro frontale con la Corona d'Aragona avrebbe comportato la logica compromissione di tutte queste delicate relazioni economiche e finanziarie. La posta in gioco era decisamente troppo grande per il non voluto possesso territoriale del Regnum Sardiniae, da cui i genovesi venivano periodica-

\footnotetext{
${ }^{26} \mathrm{C}$. MANCA, Asperti dell'espansione, cit., pp. 212-213. Va ricordato che i mercanti catalani erano esenti dal pagamento dei tributi daziari.

${ }^{27}$ Ricaviamo questi dati sempre da C. MANCA, Aspetti dell'espansione, cit., pp. 213-214, il quale nota ancora come l'attivita degli amatori liguri superi del 53\% quella dei mencanti, e cid perché i genovesi parteciparono molto attivamente come intermediari al commencio d'importazione ed esportazione tra la Sardegna e le coste italiane.

${ }^{23}$ B. GARI, La connotación estructural del conflicto entre Génova y la Corona de Aragón en el siglo XIV, in Saggi e Documenti, VI, Civico istituto colombiano, Genova, 1985, pp. 291-294.
} 
mente esclusi perché in guerra con l'Aragona, in ossequio ad una politica catalana di apertura degli scali sardi ai mercanti di nazionalità straniera ${ }^{29}$.

Sempre in merito all'interesse mostrato dai genovesi per la Sardegna e al valore da essi attribuitole, vogliamo aggiungere un'altra argomentazione. E' stato affermato che la Repubblica negli ultimi decenni del Trecento e nei primi anni del secolo successivo si mostro capace di mobilitare solo pochi esemplari di imbarcazioni per volta da inviare in soccorso dei ribelli sardi, al contrario della Corona aragonese che manifesto nello stesso periodo una capacita di mobilitazione perfino superiore a quella della prima metà del secolo ${ }^{30}$. Tale comportamento pud essere spiegato non tanto adducendo una maggiore debolezza del Comune rispetto al passato, che pure vi fu, ma un temporaneo calo d'interesse per l'isola e le sue vicende. La conferma di questa ipotesi potrebbe venire dall'impresa contro i corsari barbareschi di El Mahdia del 1390. In questa occasione in cui era in gioco la sicurezza delle sue rotte occidentali, e quindi di buona parte dei suoi traffici in uscita dal Mediterraneo, Genova seppe armare una flotta imponente: 43 galere e 8 navi, secondo i Consiglieri di Barcellona sul chi vive per paura che l'armata si dirigesse in Sardegna; 40 galere e 20 vascelli da trasporto secondo gli Annales Genuenses. Dunque una flotta in linea quantitativamente con le poderose armate organizzate per scontrarsi con $i$ catalani nel Bosforo e a Alghero a metà secolo, di dimensioni tali che in Sardegna non si vedevano ormai da quasi quarant'anni ${ }^{31}$. Volendo ora provare a rispondere al perché di tali differenze nell'impiego di mezzi economici e militari, ci viene da pensare ancora una volta, al valore attribuito alla Sardegna dagli uomini d'affari genovesi. Questa ci sembra la risposta più convincente se è vero com'è vero quanto afferma una profonda

\footnotetext{
${ }^{29}$ M. TANGHERONI, Aspetii del commercio, cit., p. 56. E' G. G. MUSso, Genovesi e Catalogno nell'ultimo medioevo: documenti d'archivio, in "Mostra documentarin LiguriaCetalogna, XII-XV secolo", Genova, 1971, pp. 41-42, a segnalare che negli enni 1386-87 vi furono catalani attivissimi a Caffa nella vita civile e nella pubblica anministrazione; rapporti questi che difficilmente si sarebbero potuti mantenere nel caso di un nuovo scontro frontale tra la Corona e la Repubblica. L'impegno da parte genovese alla composizione pecifica dei problemi era dovuto proprio alla percezione della pericolosita della situazione in Levante alla quale non bisognava aggiungere una nuova guerra con gli iberici.

${ }^{30}$ B. ANATRA, La Sardegna medioevale e modema, a cura di J. Day- B. Anatra- L. Scaraffia, Torino, 1984, p. 332.

${ }^{31} \mathrm{Cfr}$. M. T. FERRER I MALOL, Documenti casalani sulla spedizione franco-genovese in Berberia (1390), in "Miscellanea di studi storici. Collana storica di Fonti e Studi", 1, vol. I, Genova, 1969, pp. 215-225.
} 
conoscitrice della storia medioevale genovese: "non si pud parlare di politica genovese senza tenere contestualmente presente il commercio, il negotium che rappresenta l'ideale di vita collettivo e condiziona tutte le azioni e i comportamenti individuali" ${ }^{\text {32 }}$.

E proprio in virtù di questo negotium la Repubblica di Genova continuava a fornire aiuti militari ai sardi i quali non desistevano dal proseguire le loro ostilita ${ }^{33}$.

Nel mentre, tra il dicembre 1392 e il primo semestre del 1393 la Repubblica continud ad attingere grano dal regno oristanese, fino ad un ammontare di 4.206 mine pari a quasi 444.000 litri, corrispondenti al 6,5\% dei cereali da essa importati in quel periodo ${ }^{34}$.

La presenza di numerose imbarcazioni liguri negli assedi posti dai sardi alle poche roccheforti in mano agli iberici sembra indicare dalla seconda metà del 1395 un ulteriore inasprimento dei rapporti tra la Corona aragonese e la Repubblica ligure, nonostante il passaggio di quest'ultima sotto la signoria francese, con la quale il monarca aragonese intratteneva ottimi rapporti.

Le notevoli turbolenze interne del Comune e l'impegno economico e militare di Genova in favore dei sardi giudicali ebbe importanti riflessi negativi anche sulla sua economia: nel biennio 1394/1396 si produsse un notevole ribasso dei prezzi delle merci, riflesso della paura degli operatori economici liguri di una nuova imminente guerra con la Corona che avrebbe avuto effetti ancor più disastrosi sui traffici commerciali. In quegli stessi anni, inoltre, i giudicali intrattennero rapporti economici anche con mercanti di altra provenienza come dimostra la richiesta avanzata dal re Martino

\footnotetext{
${ }^{32}$ G. PETTI BALBI, Una cittd e il suo mare. Genova nel Medioevo, Bologna, 1991, p. 137.

${ }^{33}$ Il 30 dicembre 1392, avuta conferma dell'amuolamento di un grosso contingente di balestrieri - 300 o 400 - per conto di Brancaleone Doria da inviare quanto prime nell'isola insieme a due galere e una galeotta, il sovrano aragonese decise di inviare un suo ambasciatore presso il Comune italiano per intimargli di astenersi dal mantenere un simile comportamento fortemente lesivo degli accordi intercormenti. ACA, Cancilleria, Sardiniae, reg. 1943, cc. 27v28v, e Curiae sigilli secreti, reg. 1960, cc. 68r-69r.

${ }^{3} M$. Balard, La Romanie, cit., II, p. 763. Nella seconda metà dell'anno vi fu una ripresa della presenza ligure negli scali aragonesi dell'isola. Tra i mesi di luglio e novembre 1394 diversi operatori commerciali della Repubblica esportarono da Castel di Cagliari circa 1.000 litri di vino rosso e 450 di vino bianco, circa $125 \mathrm{~kg}$. di biscotto, 3 botti di pesce salato, 27 lire di cuoi e 9 lire di canapa; e vi importarono 631 lire e 17 soldi di frumento e più di 22.000 litri di vino rosso, più che mai necessari per la città affamata e privata del fertile retroterra in mano agli arborensi. ACA, Real Patrimonio, Adwanas sardas, reg. 2142, cc. 2v, $16 v-17 r, 31 r, 34 v-35 v, 107 r-v$.
} 
al sovrano castigliano perché proibisse ai suoi sudditi di recarsi a Oristano 0 in altre località sarde a lui ribelli per commerciarvi e portarvi aiuti ${ }^{35}$.

Visto la continua recrudescenza delle ostilità nell'isola e l'impossibilità di addivenire ad un accordo con il conte di Monteleone, al monarca aragonese non restò altro che dichiarare nemico e ribelle Brancaleone Doria e tutti i sardi a lui aderenti, i quali avrebbero potuto essere uccisi, danneggiati e catturati come schiavi secondo la pace a suo tempo firmata tra sardi e catalani ${ }^{36}$.

Tutto questo non significa che il rapporto tra Genova e il Giudicato d'Arborea non fosse soggetto a crisi temporanee. Il 26 gennaio 1398 al governatore francese Valerando di Lussemburgo venne chiesto di concedere alcune laudes represaliarum contro Brancaleone Doria, dominum Sardinie, e i sardi a lui soggetti i quali non avrebbero potuto più recarsi a Genova o nel suo distretto $o$ in alcun comune ad essa confederato per commerciarvi. Ancora più significativo risulta che tra coloro che chiesero tale provvedimento contro gli Arborensi figuri Giovanni dei Signori di Lagneto, più volte operante da tramite tra la potente consorteria genovese dei Doria e la famiglia regnante arborense.

Data la delicatezza del tema il governatore e il consiglio dei 15 Anziani disposero che i due richiedenti il provvedimento -il succitato Lagneto e Antonio Morando, il quale nell'ottobre 1392 dietro ordine dell'allora doge Antonio Montaldo si era recato in Sardegna per acquistarvi grano- si rivolgessero al vicario del governatore e ai "Sapientes" del Comune che poi si sarebbero consultati con loro su quanto richiesto ${ }^{37}$. Nonostante questa momentanea parentesi, il grano sardo continuava a costituire in momenti di carestie internazionali un sussidio alimentare imprescindibile per la Repubblica come risulta dimostrato da due elementi: il primo è costituito dalle norme per l'appalto del diritti dell'uno per cento gravante su tutte le merci e i beni esportate da Genova verso la Sardegna e per quelli importati dall'i-

${ }^{35}$ ACA, Cancilleria, Curie sigilli secreti, reg. 2239, cc. 12r, 54v. J. DAY, Les douanes de Gênes 1376-1377, Parigi, 1963, p. XXVII.

${ }^{36} \mathrm{ACA}$, Cancilleria, Curie sigilli secreti, reg. 2240 , cc. 32r-v. Un provvedimento simile era stato emanato anche dal fratello Giovanni I l'11 settembre 1392 sempre per la tenace opposizione dei sandi como risulta in ACA, Comune sigilli secreti, reg. 1880, ec. 130v-131r.

${ }^{37}$ ASG, Archivio segreto, Diversorum Communis Janue, reg. 3021, filza 1, doc. 174. 
sola alla città, fatta eccezione appunto per il grano e la farina ${ }^{38}$. Il secondo, invece, rappresentato dai continui acquisti cerealicoli, come avvenne il 9 marzo 1398 allorché i massari del Comune autorizzarono il pagamento di 68 lire e 4 soldi a Lodisio de Oberto per l'acquisto di una partita di 680 mine di grano sardo equivalente a 71.740 litri; due mesi più tardi, il 9 maggio 1398, per ordine del governatore francese di Genova, Sorleone Leccavello e socio, massari del Comune, dovevano pagare a Nicola Becchignone olim Centurione 439 lire e 8 soldi per un carico di ben 4.394 mine di grano, pari a circa 463.567 litri, portato dalla Sardegna ${ }^{39}$.

$\mathrm{Ma}$, come sua abitudine, Genova mentre da un lato continuava a mantenere stretti contatti con i sardi giudicali dall'altro non poteva fare a meno di valutare con attenzione i rapporti con la Corona iberica nella loro interezza, i quali subirono un deciso peggioramento e spinsero il 13 maggio 1398 il governatore francese Borleo di Lussemburgo, considerata la reciproca utilità, ad intavolare trattative per giungere alla cessazione delle ostilità da ambo le parti con reciproco impegno al mantenimento di buone relazioni nei rispettivi dominî ${ }^{40}$.

Circa due settimane più tardi venne formulata la decisione del Comune ligure in merito alla richiesta di lettere di rappresaglia contro Brancaleone Doria e i sardi: il 30 maggio il vescovo di Meaux, nuovo

\footnotetext{
${ }^{38}$ Il documento in questione, del 1398, riporta chiaramente le merci per cui si doveva esigere il pagamento del tributo: "perlarum veratium, lapidum preciosorum, pannorum lane, lini, sette ..., exceptis semper grano et farina e quibus et pro quibus non solvatur predictus introitus". Cfr. Liber institutionuin cabellaruin veteruin, a cura di D. Gioff rè, coll. Acta italica, 12, Milano, 1967, pp. 186-187.

${ }^{39}$ ASG, Antico Comune, Magistrorum rationaliuın appodixiae, reg. 104, c. 23r. In un'altra serie dell'archivio genovese abbiamo rinvenuto anche il mandato di pagamento emanato dalla Massaria del Comune a favore del succitato Lodisio de Oberto il 5 maggio dello stesso anno, e un altro a favore di Niccolò Centurione olim Becchignone in date 17 e 22 maggio. ASG, Antico Comune, Commnunis Janue massaria, reg. 24, cc. 41v, 43r, 168v; magistrorum rationalium appodixiae, reg. 104, c. 21v. Per spiegare qucsto rinnovato interesse di Genova per il mercato cerealicolo arborense si devono considerare due elementi: da una parte i dati sulla flessione delle esportazioni cerealicole siciliane riportati da P. CORRAO, Mercanti veneziani ed economia siciliana alla fine del XVV secolo, in "Medioevo.Saggi e Rassegne", 6, 1981 , p. 140, e dall'altra anche la proibizione emanata nel gennaio dello stesso anno dal re Giovanni I di concedere licenze di esportazione di cereali dai territori della sua Corona verso la Repubblica tirrenica, considerata la seria carestia che rischiava di ripercuotersi sulla stessa Barcellona, efr. Archivo Historico de la Ciudad de Barcelona, Consell de Cent, Llibre del Consell, reg. $1-27$, c. $138 \mathrm{v}$.
}

${ }^{40}$ ASG, Archivio segreto, Diversorum negociorum cancellarie Communis Janue, reg. 498, cc. 129r-v. Le negoziazioni diplomatiche si rivelarono lunghe e difficili, al punto da sfociare in un rinnovo di pace tra i due stati solo cinque anni più tardi. 
governatore di Genova, proibl ai cittadini del Comune e del suo distretto di commerciare e di formare società con il conte di Monteleone o con i suoi sudditi ${ }^{41}$.

Il provvedimento era estremamente pericoloso per il regno sardo perché lo privava dell'unico appoggio internazionale di un certo spessore di cui godeva e sul cui aiuto aveva sempre potuto contare fin dall'inizio delle ostilità con la Corona d'Aragona, e perché la circostanza che a richiedere le laudes di rappresaglia fosse stato Giovanni dei Signori di Lagneto, strettamente legato ai Doria genovesi e allo stesso conte di Monteleone ci permette di ipotizzare una momentanea frattura tra i rami sardo e genovese della consorteria Doria che si dovette pero risolvere gia l'anno successivo. Cosl almeno crediamo che faccia intendere la nomina del mercante Branca Doria del fu Enrichetto a console dei genovesi a Oristano effettuata il 16 ottobre 1399, segno inequivocabile di una notevole intensità e regolarità dei traffici commerciali che in quegli anni univano il Giudicato d'Arborea alla Repubblica di Genova ${ }^{42}$

Nonostante che negli ultimissimi anni del Trecento la Superba avesse ulteriormente aumentato le proprie azioni militari in appoggio dei giudicali, le trattative diplomatiche con la corona d'Aragona per il rinnovo della pace proseguirono, al pari di quelle con i sardi giudicali come testimonia la concessione nell'estate del 1398 di una tregua valevole tre mesi. Anche per questo agli inizi del Quattrocento le ostilità proseguirono abbastanza stancamente. Peraltro, a rendere ancora più precaria la situazione sarda si aggiunse l'ennesima epidemia di peste che colpl l'isola nel 1402 , e che con ogni probabilità tra la fine del mese di maggio e i primi 23 giorni di giugno del 1403 causo la morte di Eleonora d'Arborea ${ }^{43}$.

\footnotetext{
${ }^{4}$ ASG, Archivio segreto, Diversorum negociorum cancellarie Communis Janue, reg. 498 , c. $147 \mathrm{r}-\mathrm{v}$.

${ }^{42}$ ASG, Archivio segreto, Diversorum negociorum cancellerie Communis Janue, reg. 499, c. 152. G. BANDINI, Lettere datiniane pervenute dalla Sardegna, in "Annali della Faccoltà di Economie e Commercio dell'Universitâ di Cagliari", 1959-60, pp. 8-9, servendosi di alcune lettere dell'archivio della Compagnia Datini, evidenzia come la città di Oristano tra gli anni 1391-1401 fosse ancora un mercalo interessante da dove si potevano esportare cuoi, pelli, sego, formaggi $e$ in cui si potevano importare panni, armi, coltclleria, vasellame di Firenze. Né sfugge alla lettura delle stesse fonti come al contrurio l'isolamento del Regnum avesse reso la sua economia non molto fiorente.
}

${ }^{43}$ ACA, Cancilleria, Curie sigilli secreti , reg. 2240, c. 124r-v. L. GaLUnNARI, Sulla data di morte di Eleonora di Aiborea, in "Mediocvo. Saggi c Rasscgne", 19, in corso di stampa. 
Le negoziazioni diplomatiche tra la Corona e la Repubblica risultarono lunghe e difficili, testimoniate da numerosi documenti che evidenziano come il nodo dei problemi tra i due contendenti fosse proprio l'appoggio dato dai liguri ai sardi ribellit4. Il raggiungimento dell'accordo fu costellato da diversi incidenti di attacchi genovesi a imbarcazioni catalane, che spinsero il 28 luglio 1400 il sovrano ad ordinare l'arresto dei liguri residenti nei suoi regni di Valenza e Maiorca

e il sequestro dei loro beni ${ }^{45}$. Nonostante cio, alcuni mesi più tardi, nel febbraio 1401, al fine di non compromettere definitivamente il tentativo di composizione diplomatica con Genova, Martino l'Umano non volle acconsentire alle numerose richieste avanzategli dai suoi sudditi di aumentare le tasse ai cittadini liguri residenti nella sua signoria, dando come ulteriore prova di buona volontà la restituzione a Degerino de Podio, ambasciatore del Comune ligure, di alcune merci depredate a mercanti genovesi dai suoi sudditi. Ulteriore tempo passò per la scelta da parte catalana dei due procuratori che avrebbero dovuto rappresentare il sovrano nell'incontro di Marsiglia con gli inviati di Genova per la ratifica del trattato ${ }^{46}$. Nuovi ritardi si verificarono anche per il sopraggiungere di problemi tra l'Aragona e il finitimo regno di Navarra che richiesero la partecipazione personale dello stesso monarca, come egli spiego al governatore francese di Genova in una lettera datata 24 febbraio 1402 al quale garantl che i suoi procuratori si sarebbero trovati a Marsiglia per il futuro mese di maggio. Ma neanche questa volta i tempi dell'accordo furono rispettati: sei mesi più tardi Martino si trovava ancora alle prese con il punto nodale dei rapporti con il Comune ligure: la Sardegna; il sovrano, infatti, ribadl ai suoi due emissari la propria fermezza nel voler impedire ai genovesi l'accesso ai territori sardi a lui ribelli, secondo quanto stabilito dalla pace voluta da suo padre, e nel

\footnotetext{
"ACA, Cancillerla, Curie sigilli secreti, reg. 2240, cc. 139v-140r; reg. 2242 cc. 78v80v, 94r; reg. 2243, cc. 5r-6r.

${ }^{45} \mathrm{ACA}$, Cancillerla, Curie sigilli secreti, reg. 2243, c. $122 \mathrm{v}$ e 183 v., quest'ultimo gia citato da M.T. FERRER I MAWOL, Els italians a terres catalanes, in "Anuario de Estudios Medievales", 10 (1980), p. 439.

${ }^{46} \mathrm{ACA}$, Cancillerla, Curie sigilli secreti, reg. 2244, cc. 1v-2r, 14r-v, 30r-v, 32r, 70r-v. In data 4 settembre 1401 il sovrano informo Percivale Vivaldi, console dei Catalani a Genova, dal quale aveva saputo che la Repubblica aveva nominato come suoi rappresentanti Damiano Cattaneo e Antonio Re, che la scelta dei suoi procuratori era caduta su Francesc Fluvid e Pere ça Calm.
} 
voler garantire loro al contrario un sicuro accesso nelle località isolane sotto il suo dominio ${ }^{47}$.

L'aleatorietà della situazione appare confermata anche dalle fonti genovesi,dalle quali risulta che nel 1402 la Superba poté importare dall'Arborea solo 332 mine di grano (=ca. 35.000 litri) pari all' $1 \%$ del mercato di importazione cerealicolo genovese di quell'anno ${ }^{48}$.

Mentre tra sardi e catalani e tra questi ultimi e i genovesi si cercava di raggiungere nuovi accordi che permettessero a ciascuno dei contendenti di riprendere fiato, la Repubblica ligure non desisteva dal realizzare azioni navali che, seppure formalmente dirette contro pirati e corsari, in pratica risultavano di sostegno ai sardi arborensi.

E' dell'aprile 1402 un interessante registro della serie Galearum marinariorum rationes dell'archivio genovese che conserva le spese sostenute per l'arruolamento di marinai e balestrieri di una galera ad custodiam maris patronizzata "per nobilem dominum Andream Lomellinum ad custodiam Provincie, Corsice et Sardinie pro inquirendo piratarum vel malle hobedientes Comunis Ianue", che dovette operare nei mesi successivi all'aprile del $1402^{49}$.

Ancora una volta, pero, proprio mentre andava aumentando la propria azione militare a sostegno dei sardi giudicali, la Repubblica ligure, vista l'imminenza del nuovo scontro con Venezia voluto dal governatore Boucicaut, il 13 gennaio 1403 riuscl a rinnovare l'ennesimo trattato di pace con la Corona d'Aragona, il quale riprese i termini dell'accordo firmato nel 1390 , a dimostrazione che la via diplomatica più volte tentata dai due stati

${ }^{47} \mathrm{ACA}$, Cancilleria, Curie sigilli secreti, reg. 2244, c. 135r-v, reg. 2245 c. 28r-v. Oltre a cio il sovrano per quanto riguardava invece Alghero e Bonifacio dispose che non vi fosse alcuna innovazione.

${ }^{48} \mathrm{Cfr}$. M. BALARD, La Romanie, cit., II, p. 764. Per la politica di approvvigionamento cerealicolo nel XV secolo rimandiamo a J. HEERS, Genova, cit., pp. 201-216.

${ }^{49}$ ASG, Antico Comune, Galearuin marinariorum rationes, n. 662. Andrea Lomellini, membro dell'omonima famiglia che proprio in questi anni ebbe sotto il suo controllo quasi tutta la Maona di Corsica, per via di un fratello - Lionello, sposato con Pietra Doria - era imparentato con il potente nucleo familiare sardo-genovese. Né si possono trascurare i suoi forti legami con il govematore Boucicaut al quale fu fedele tutta la famiglia Lomellini come pure anche la Doria, sia pure dopo un iniziale momento di ostilità. Cfr. G. PETT BALBI, Una cittd, cit., p.p. 230-235. ASG, Antico Comune, Magistrorum rationalium sententiae, reg. 88, cc. $30 v-31 \mathrm{r}$. 
per la composizione delle reciproche divergenze non era in grado di eliminare definitivamente i punti di attrito ${ }^{50}$.

Lo dimostra il fatto che nuovi incontri diplomatici avvennero anche l'anno successivo. Il 3 e 4 maggio 1404 nei registri contabili della Massaria del Comune di Genova vennero annotate le somme dirette a Antonio Cattaneo e Tommaso de Fornariis, protectores pacis Catalanorum, per coprire le spese per l'invio del notaio Bartolino Sisto ituro ad serenissimum dominum regem Aragonum. L'anno successivo venne inviata una nuova ambasceria guidata da Antonio Re, procuratore del Comune ligure durante la stipula del precedente accordo firmato due anni prima, insieme al legum doctor Ingo Grimaldi. L'ambasceria ebbe inizio il 6 dicembre 1404 quando gli inviati partirono da Genova e termino il 18 aprile 1405 quando Antonio $\mathrm{Re}$ e $\mathrm{i}$ tre famigli che lo avevano accompagnato fecero ritorno in patria ${ }^{51}$.

Anche questa nuova pace dovette risultare aleatoria come provano i successivi attacchi navali della Repubblica portati soprattutto alle imbarcazioni catalane tra l'isola dell'Asinara e Longosardo, all'interno di un nuovo interesse ligure anche per la Corsica, in un disegno globale mirante a eliminare in via definitiva la presenza catalano-aragonese dal Tirreno ${ }^{52}$.

\footnotetext{
${ }^{50}$ A BoRland, Janua, cit., p. 17. Il testo del trattato di pace firmato il 13 gennaio 1403 $z$ in Colección de documentos inéditos del Archivo de la Corona d'Aragón, (d'ora in avanti Codoin) a cura di P. de Bofarull i Mascano, vol. I Tomo I, Barcellona, 1847, pp. 1-24; di esso si occupd anche A. BOSCOLO, La politica italiana di Martino il Vecchio re d'Aragona, Padova, 1962, pp. 64-65, e J. CAMAREnA MAHIQUES, Tratado de paz entre Aragón y Génova en 1413, Valenza, 1952, p. 96, il quale osserva che particolare attenzione fu dedicata dalle due parti in particolar modo all'indennizzo dei danni causati dai ripetuti scontri avvenuti tra le rispettive imbarcazioni soprattutto in acque sarde a partire dal 1390 data della stipula dell'ultimo accordo tra $\mathrm{i}$ due Stati. In realta subito all'indomani del rinnovo del trattato di pace si verificarono pericolosi incidenti tra genovesi e catalani che parvero mettere a repentaglio l'accordo appena raggiunto. ACA, Cancilleria, Curie sigilli secreti, reg. 2245, cc. 141r, 170rv, reg. 2246, cc. 57v-58r; AHCB, Consell de Cent, Llibre del Consell, reg. I-28, c. 54r-v.

${ }^{31}$ I conti riguardanti il notaio Bartolino Sisto sono conservati in ASG, Antico Comune, Communis Janue Massaria, reg. 29, cc. 3r, 44v, 49r, 88 e 96; quelli pertinenti invece l'ambasceria di Antonio Re sono nella stessa sezione dell'archivio genovese, nel reg. 30 a cc. $3 r$, 49r e nel reg. 31 a c. $115(7 v)$.

${ }^{52}$ Cfr. G. PeTn BALBI, Una cittd, cit., pp. 241-242. M. BALARD, La Romanie, cit., II, p. 764 , calcola che nel 1405 dall'isola partirono alla volta della Repubblica 2.040 mine di cereali, pari a poco più di 215.000 litri, costituenti il $6 \%$ dell'intero mercato genovese di rifomimento cerealicolo. L' 11 maggio 1405 il re Martino rispose a Vincentello d'Istria di essere rimasto meravigliato per aver saputo che i Bonificacini, usando la bandiera del Comune di Genova, con alcune imbarcazioni a remi avessero attaccato diverse località corse sotto l'autorità regia uccidendo e danneggiando i sudditi a lui fedeli; considerata la situazione il sovrano autorizzd il detto Vincentello a difendersi dagli attacchi portati contro i corsi a lui soggetti senza però arrecare danni a Calvi e Bonifacio prima di aver ricevuto una risposta in merito dal governatore del Comune per non infrangere la pace. ACA, Cancilleria, Curiae sigilli secreti,
} 
Per quanto attiene alla Sardegna, la conferma di questo brusco peggioramento della situazione viene data da alcuni documenti emanati negli ultimi tre mesi del 1405 dal sovrano aragonese, riguardanti la missione di Joan de Vallterra, da lui inviato nell'isola per trattare con Brancaleone Doria una nuova pace, dai quali trapela la convinzione del sovrano che il conte di Monteleone non avesse una reale volontà di addivenire ad un accordo $^{53}$.

Pressoché in contemporanea si registro un aumento della tensione anche nelle relazioni tra Genova e l'Aragona, testimoniata ancora una volta dall'armamento di una galera, patronizzata da Pietro Doria, che opero nell'estate del 1407 nelle acque sarde riuscendo a catturare quattro famosi pirati catalanis ${ }^{4}$. Ancora una volta dunque a guidare la spedizione in soccorso dei sardi giudicali era stato scelto un membro della famiglia Doria, segno che per il momento l'alleanza tra il ramo sardo e quello ligure della consorteria era ancora operante, cosl come quello ancora più importante tra i Doria genovesi e il governatore Boucicaut, che rendeva possibile l'invio nell'isola di continui contingenti d'armati ed imbarcazioni.

A rendere pero più complessa la situazione sarda fu la morte nel 1407 senza eredi di Mariano V, figlio di Brancaleone e Eleonora e ultimo discendente della casata oristanese.

In concomitanza di cio nell'estate di quell'anno si assistette ad una escalation notevole dell'intervento navale genovese: il 18 luglio vennero inviate al sovrano aragonese notizie dettagliate sulla situazione dell'isola; una particolare attenzione venne dedicata a Longosardo e al porto di Ullastre, nella

reg. 2248 , c. $110 \mathrm{r}-\mathrm{v}$.

${ }^{33}$ ACA, Cancilleria, Curie sigilli secreti, reg. 2248 c. $165 \mathrm{r}$, in data 19 settembre 1405, indirizzato al suo emissario Vallterra il quale ricevette l'istruzione di fermarai in Sardegna per qualche tempo ancora in vista di un improbabile accordo; reg. 2249 c. $32 \mathrm{r}-\mathrm{v}$ emanato il 22 dicembre dello stesso anno e contenente l'ordine per il Vallterra di far ritomo in Catalogna visto il fallimento delle trattative con il Doria.

${ }^{44}$ ASG, Antico Comune, Marinariorum galearum rationes, reg. 667, composto al momento dell'arruolamento dell'equipaggio, e reg. 665 redatto invece a viaggio concluso. Ulteriore documentaziono attestante le diverse voci di spesa affrontate dal Comune di Genova per dotare l'imbarcazione di Pietro Doria non solo di equipaggio ma anche di vettovaglie, ami, calafataggio ecc. d stata rinvenuta in ASG, Archivio Segreto, Officium Baylie, reg. 711, cc. 9v-12r, $14 r-15 r, 17 v-18 r, 23 r-25 r, 28 r, 35 v-42 r, 47 v$, $51 r-v$ e $53 r$. Sempro in merito alla opodizione guidata da Pietro Doria vedasi quanto dicono STELLE G. ET I., Annales genuenses, in Rerum Italicarum Scriptores, XVII, p. 2, a cura di G. Petti Balbi, Bologna, 1975, p. 281. 
costa orientale, assediato e danneggiato da 2 navi e 2 galere genovesis ${ }^{35}$. Le imbarcazioni liguri cercavano di realizzare un blocco dei collegamenti con la penisola iberica, nella speranza di riuscire a far capitolare le uniche roccheforti ancora in mano dei catalano-aragonesi.

Nell'estate dell'anno successivo, il 1408, incrocio per quattro mesi i mari sardi anche la galera ad custodiam maris del Comune di Genova sotto la guida di Battista Montaldo, il quale durante il pattugliamento dell' $i$ sola ebbe modo di catturare sette pirati catalani e castigliani, poi condotti a Genova dove vennero impiccatit ${ }^{36}$.

Nel frattempo in Arborea la Corona de Logu nomind nuovo giudice Guglielmo II visconte di Narbona, nipote di Beatrice d'Arborea, sorella di Eleonora, e, in sua attesa, come vicario Leonardo Cubello, figlio di un cugino della defunta giudicessa ${ }^{57}$.

I legami personali e politici che univano Guglielmo II a Genova, dove governava l'amico Boucicaut, e a Napoli dove regnava Ladislao d'Angio-Durazzo, tutti oppositori della Corona d'Aragona, sulla carta sembravano promettere positivi riflessi sull'esito della lunga guerra.

Sul fronte interno, pero, questa scelta comporto l'uscita di scena definitiva di Brancaleone Doria, estromesso dalla gestione politica del giudicato dal visconte e imprigionato fino alla morte a Bosa ${ }^{58}$. Episodio che a detta dei coevi annalisti genovesi non si sarebbe mai verificato "si Ianuensium et suorum de Auria elegisset manere consilio". Genova aveva deciso di continuare ad appoggiare la causa sarda, schierandosi pero dalla parte di Guglielmo III, legittimo erede e successore al trono oristanese. Non vanno dimenticati l'amicizia e i vincoli politici passanti per la Corte di Francia che univano Boucicaut al visconte di Narbona; né si puo tralasciare che la famiglia Doria ben difficilmente avrebbe incrinato l'importante

\footnotetext{
${ }^{5 s}$ Archivio di Stato di Cagliari (d'ora in avanti ASC), Antico Archivio Regio, Luogotenenza generale, vol. K 3, c. 12 v.

${ }^{36}$ ASG, Antico Comune, Galearum marinariorum rationes, regg. 668 e 670 . Cfr. anche STELLAE G. ET., Annales genuenses, cit., p. 284. Sempre in ASG, Antico Comune, nelle serie Communis Janue Massaria, reg. 32, e Magistrorum rationalium appodixiae, reg. 107 abbiamo rinvenuto $\mathrm{i}$ conti delle spese sostenute dal Comune di Genova per l'amamento della galera di Battista Montaldo, per il suo stipendio e per i lavori di inanutenzione dell'imbarcazione.

${ }^{57}$ Per inaggiori dettagli sulla lunga e complessa fase della successione sul trono giudicale vedasi F. C. CasULA, La Sardegna, cit., II, pp. 504-507.

${ }^{80} \mathrm{Cfr}$. LUCIANO GALUNARI, Guglielmo III di Narbona, ultimo sovrano di Arborea, e la guerra dei Cent'Anni, in "Medioevo. Saggi e Rassegne", 18, 1993, pp. 96-98.
} 
rapporto con il governatore francese per sostenere ancora i piani di Brancaleone Doria deciso a contrastare con le armi Guglielmo III ${ }^{\text {s9 }}$.

A questo punto, eliminato dalla scena politica sarda il conte di Monteleone, l'unico interlocutore dei catalani e dei genovesi rimase $\mathrm{Gu}$ glielmo III il quale continud a ricevere aiuti e rifornimenti da Genova: 1'8 maggio 1409 Niccold Panizzario di Sestri Ponente riconobbe davanti al notaio Giuliano Canella di aver acquistato una partita di spade che avrebbe pagato al suo ritorno dalla Sardegna; lo stesso giorno e dinanzi al medesimo notaio il cittadino di Genova Marco de Mari dichiaro di possedere due dei ventiquattro carati (o parti) della nave di Francesco Spinola che entro breve tempo sarebbe passata in Sardegna insieme ad'una armata che vi si doveva recare ${ }^{60}$.

La Corona temeva soprattutto un forte invio in Sardegna di soldati e balestrieri sotto la guida dello stesso Boucicaut: "[el] senyor [Martino il Vecchio] ha sabut certament que en Jenova se són armades e.s'armen certes naus e galeas que deven passar en Çardenya ab gens d'armes e ballesters, dels quals se.n diu que serà capitá mossèn Bouciquaut per ajudar al dit vezcomte de Narbona". Altre preoccupazioni per il sovrano aragonese venivano dai soccorsi per Guglielmo III preparati in Francia con il beneplacito dello stesso Carlo VI: "E que en lo regne de França se fan molts altres preparatoris per ajudar e trametre seccors al dit vezcomte, de que lo dit senyor [il re d'Aragona] és fort mervellat qar no és presumidor que semblants coses se gosassen asseiar ne portar a conclusió sens special licencia e voler seu [del re di Francia]"61.

Subito dopo la disfatta arborense avvenuta a Sanluri il 30 giugno 1409 il ramo sardo della famiglia Doria, ormai allontanato definitivamente

${ }^{59}$ STELLAE G. ET I., Annales genuenses, cit., p. 286.

${ }^{60}$ ASG, Antichi notai, Giuliano Canella, n. 497/I, cc. 6v-7r e 37r-v. Sempre nel mese di maggio 1409 vi si recarono Tommaso Savignone, Bemabò Dentuto, Nicolò Andrea Lomellini e infine Francesco Spinola, citato anche nel documento notarile da noi rinvenuto. Cfr. M. T. FERRER I MALLOL, Dos registres de l' "Officium maris" di Genova (1402-1403; 1408-1410), in "Atti del I congresso storico Liguria-Calalogna" (14/19 ottobre 1969), Bordighera, 1974, pp. 249-272.

${ }^{61}$ Il memoriale dell'ambasciata inviata da Martino l'Uınano a Carlo VI di Francia 2 in ACA, Cancilleria, reg. 2252, cc. 77r-78v, già citato da F. C. CASULA, La Sardegna aragonese, II, cit., pp. 517-519. e soprattutto p. 723. Esso si chiudeva con i ringraziamenti del sovrano aragonese a Luigi II d'Angid per non aver permesso ai suoi sudditi di recarsi in Sardegna in aiuto del visconte. Cfr. anche F. C. CASULA, Ricerche archivistiche sulla battaglia di Sanluri del 1409, "Archivio Storico Sardo", XXXV, 1986, p. 130. 
dal trono oristanese, sembro muoversi in maniera più defilata, mentre fu ancora Guglielmo III, desideroso di recuperare le posizioni perdute, a ricorrere all'aiuto dell'amico Boucicaut, il quale pero era giunto alla fine della sua avventura genovese ${ }^{62}$.

Considerato momentaneamente chiuso il discorso con Genova, passata sotto la dominazione del marchese di Monferrato, al visconte non restava altro da fare che procurarsi gli aiuti necessari in Francia, dove pero la situazione bellica era tornata a farsi preoccupante per la ripresa delle attività militari inglesi ${ }^{63}$.

Il che non significa che la Repubblica smettesse di occuparsi della Sardegna; indubbiamente, pero, il fatto che il Giudicato fosse stato smembrato e che il ramo sardo della famiglia Doria non avesse più gli stretti legami del passato con il trono oristanese modifico non poco la natura degli interventi liguri nell'isola. Dal 1410 in poi Genova continuo a contendere all'Aragona il dominio del mar Tirreno; anzi, negli anni successivi la sua azione ando aumentando di intensita, pur essendo più autonoma da quanto si decideva in contemporanea in cio che restava del Giudicato di Arborea ormai avviato alla definitiva scomparsa.

\section{RÉSUMÉ}

A la lumiere de nouveaux documents des Archives de la Couronne d'Aragon de Barcelone et des Archives de l'État de Gênes, cet article présente un nouveau tableau des rélations politiques et économiques entre le

\footnotetext{
${ }^{62}$ STELLAE G. ET I., Annales genuenses, cit., p. 293. Su Jean le Meingre e la sua politica vedansi almeno F. SURDICH, Genova e Venezia, cit.; J. E. RUIZ DOMENFC, Boucicaut, gobemador de Génova. Biografia de un caballero errante, Civico istituto colombiano. Studi e testi. 12, a cura di G. Pistarino, Genova, 1989, e ID., Boucicaut o come riconoscere l'altro, in La Storia dei genovesi, "Atti del Convegno di studi sui ceti dirigenti nelle istituzioni della Repubblica di Genova" (Genova 8/10 giugno 1988), IX, Genova, 1989, pp. 311-318. Anche J. HEERS, Boucicaut et la rébellion de Gênes (1409-1410): armée royale, armée princiere o partisane?, in La Storia dei genovesi, cit., XI, Genova, 1991, pp. 43-63.

${ }^{63}$ Sugli avvenimenti occorsi in Francia in questi anni vedansi almeno $\mathrm{CH}_{\mathrm{H}}$. ALJMAND, La guerra dei Cent'Anni, Milano, 1990, F. AUTRAND, Charles VI, 1986, J. FAvite, La guerre de Cent Ans, Parigi, 1980, B. SChNERB, Les Armagnacs et les Bourguignons. La maudite guerre, Parigi, 1988.
} 
Judicat ou royaume d'Arborea, la République de Gênes et la Couronne d'Aragon pendant les années des règnes des souverains aragonais Jean I, Martin l'Humain, Ferdinand I et les premières années d'Alfons V (13871420), qui voyèrent la fin de l'expérience d'état du dernier judicat sarde.

Les documents inédits génois et aragonais permettent de mettre en rélief surtout la rôle joué par la Couronne française, détentrice de la domination sur Gênes, dans l'échiquier méditerranéen de ce temps la.

\section{SUMMARY}

In the light of new documents that have been found in the Archivo de la Corona de Aragon in Barcelona and in the Archivio di Stato of Genoa, this article presents a new outline of the political and economic relationship among the "Giudicato" (Kingdom) of Arborea, the Republic of Genoa and the Crown of Aragon during the reigns of John I, Martin the Human, Ferdinand I and the first years of the reign of Alfonso V (13871420), a period during which took place the end of the state experience of the last Sardinian "Giudicato".

These unpublished Genoese and Aragonese documents allow us to point out especially the role played by the French Crown, holder of the lordship of Genoa, in the Mediterranean area. 